\title{
The diffusion process of patient education in Dutch community pharmacy: an exploration
}

\author{
M.C.M. Pronk ${ }^{\mathrm{a}, *}$, A.Th.G. Blom ${ }^{\mathrm{a}}$, R. Jonkers ${ }^{\mathrm{b}}$, A. Van Burg ${ }^{\mathrm{c}}$ \\ ${ }^{\mathrm{a}}$ Department of Pharmacoepidemiology and Pharmacotherapy, Utrecht Institute for Pharmaceutical Sciences (UIPS), PO Box 80082, \\ 3508 TB Utrecht, The Netherlands \\ ${ }^{\mathrm{b}}$ ResCon, Haarlem, The Netherlands \\ ${ }^{\mathrm{c}}$ Netherlands Institute for Health Promotion and Disease Prevention (NIGZ), Woerden, The Netherlands
}

Received 12 March 1999; received in revised form 10 December 1999; accepted 4 January 2000

\begin{abstract}
Patient education activities in pharmacies are receiving much attention. These activities are relatively new and implementation requires individual and organisational change in pharmacies. The aim of this study is to identify barriers and facilitators to the implementation of patient education in community pharmacies and to classify these barriers and facilitators into the diffusion stages of Rogers' 'Innovations in Organisations' model [Rogers, EM. Diffusion of innovations. 4th ed. New York: The Free Press, 1995]. Six focus group interviews, three with pharmacists and three with pharmacy technicians (total $n=38$ ) were carried out. The initiation phase has been dealt with by community pharmacies, whereas the implementation phase has not. The barriers and facilitators in the redefining/restructuring stage were mainly related to the organisation of patient education. In the clarifying and routinizing stages, barriers were related to repetition and knowledge transfer. The facilitators in these stages relate to performing and talking about patient education. Interventions for implementation of patient education should aim at these barriers and facilitating factors. (C) 2001 Elsevier Science Ireland Ltd. All rights reserved.
\end{abstract}

Keywords: Innovations in organisations; Community pharmacy; Patient education

\section{Introduction}

During the past 30 years changes have occurred with respect to community pharmacy practice in the Netherlands. Activities have changed from product-

\footnotetext{
*Corresponding author. Tel.: + 31-30-253-6966; fax: + 31-30253-9166.

E-mail address: m.c.m.pronk@pharm.uu.nl (M.C.M. Pronk).
}

centred to patient-centred. The emphasis used to be on preparing drugs, whereas nowadays only about $5 \%$ of the medication are prepared in community pharmacy. The communication with patients, especially patient education, has become much more important [2,3].

In 1998, a community pharmacy in The Netherlands served an average of 9000 customers. The average staff was 1.54 pharmacists and 5.79 techni- 
cians (converted to full-time equivalents). Most patients receive their prescription drugs at one of the 1547 community pharmacies, although in rural areas prescription drugs are dispensed by about 620 physicians (in 1998) [4]. Pharmacists and pharmacy technicians may both be involved in patient education, but technicians are involved in the majority of the contacts [5].

Within the pharmacy profession, patient education was defined as: 'the communicative activities addressed to the patient/client, with the main objective to realise patients' proper drug use' [5].

Other new tasks are medication surveillance (control for the right dosage, interactions between drugs, contra-indications and adherence to the drug regimen) and informing prescribing physicians and other health workers about drugs [3].

Pharmaceutical care is the concept that covers these kinds of activities. Pharmaceutical care has been defined as: The adequate provision of drug therapy for the purpose of achieving definite outcomes that improve a patient's quality of life [6]. In the literature on pharmaceutical care, patient education activities and patient counseling often are included [7].

Pharmacies are experiencing difficulties with adjusting to and implementing of their new task of patient education. Therefore, a quasi-experimental study on improving the implementation of patient education was started, with emphasis on organisational aspects like work process and preconditions. The first step in the development of our intervention for the implementation of patient education consisted of an explorative analysis of the barriers and facilitators regarding the implementation of patient education in Dutch community pharmacy. Implementing patient education involves an organisational and individual change process, for the description of which Rogers' 'Innovations in Organisations' model is useful as a framework [1].

The main purpose of this article is to classify the reported barriers and facilitators with respect to implementation of patient education into the diffusion stages of Rogers, and therefore to indicate answers to the following questions: (1) What are barriers and facilitators for implementation of patient education in Dutch community pharmacy, as experienced by pharmacists and pharmacy technicians?
(2) In what stage of Rogers' 'Innovations in Organisations' model can these barriers and facilitators be classified?

To show how far community pharmacies have proceeded in this process and to understand why we focussed on the implementation of patient education, we will describe the model for the diffusion process of patient education in community pharmacy [1].

\subsection{Rogers' innovations in organisations model}

Rogers' Innovations in Organisations model describes the process organisations can go through before they adopt and integrate innovations into daily practice. It consists of five stages, the first two in the initiation subprocess and the latter three in the implementation subprocess [1]. The stages a community pharmacy can go through when implementing patient education are illustrated here.

\subsubsection{Agenda setting}

Agenda setting occurs when a problem is defined that may create a perceived need for an innovation. Nowadays, an important mission of community pharmacies is to adequately provide drug therapy for achieving outcomes that improve a patient's quality of life. Communication with patients is an essential part of adequate drug provision and it was set on the agenda of pharmacists from the 1970s onwards $[7,8]$. This was also influenced by legislation $[9,10]$ and non-compliance of patients with their medication regimen [5,11]. Patient education has also been a subject in a large amount of issues of pharmacists' and pharmacy technicians' professional journals [10,12-14]. The positive attitudes of pharmacists and pharmacy technicians towards patient education may also reveal that patient education is on the agenda of pharmacies $[2,15,16]$.

\subsubsection{Matching}

In the matching stage, a problem from the organisation's agenda is fit with an innovation. This means that problems with providing good pharmaceutical care are fit with patient education, and this match is planned and designed. In this stage the organisation's members attempt to determine the feasibility of the innovation. Conceptual matching of patient education activities with the work process in the pharmacy 
can be seen in standards and guidelines that were developed $[8,17]$. These tools are operationalisations of the concept of patient education for community pharmacy practice. The initiation phase ends with the decision to adopt the innovation, which pharmacists have done by saying 'yes' to providing patient education [15].

\subsubsection{Redefining/restructuring}

The actual performance of the innovation takes place in the implementation subprocess, which consists of the redefining/restructuring, the clarifying and the routinizing stage [1].

In the redefining/restructuring stage, both the organisation and the innovation are adjusted in such a way that the innovation fits within the organisation. In community pharmacy this includes a redefinition of patient education: 'What exactly do we mean by patient education?' and a restructuring of the pharmacy: 'How should we adjust the pharmacy and the pharmacy work process to facilitate patient education activities?'

Several studies indicate barriers to providing patient education, such as time constraints, workload and lack of privacy in the pharmacy [18-22]. These barriers can be considered to be indicators for lack of adjustment, either lack of redefining or lack of restructuring of the work process in the pharmacy.

\subsubsection{Clarifying}

The clarifying stage of the innovation process occurs when the innovation is put into use in the organisation, so that the meaning becomes clearer to the members of the organisation and uncertainty is reduced through a process of talking about the innovation and trying it out.

It is not clear whether community pharmacies are in this stage. Reports or activities that could be indicative for this stage were not found.

\subsubsection{Routinizing}

Routinizing occurs when the innovation has become a regular activity of the organisation and has lost its separate identity. The innovation is absorbed by the organisation's ongoing activities. At that stage the implementation is complete. Has community pharmacy reached this stage? As long as barriers like time, workload and lack of privacy exist (Section 1.1.3), probably not.

Up to the redefining/restructuring stage indicators were found for pharmacies to have dealt with the stages or to be working on them. Indicators for clarifying and routinizing were not found. Therefore, the focus group interviews in this study are used to explore facilitators and barriers in the implementation subprocess.

\section{Methods}

To explore the barriers and facilitators with respect to implementation of patient education in Dutch community pharmacy, focus group interviews were carried out on a regional level (north, centre and south of the Netherlands) with pharmacists and technicians [23,24]. The interviews were semi-structured, which means that there was a framework of open questions that had to be asked. Among others, the following questions were asked:

1. Which factors stimulate the implementation of patient education in community pharmacies? (facilitators)

2. What can be improved with regard to the implementation of patient education? (barriers)

The names and addresses of 90 technicians, 30 from each region were randomly obtained from a database of technicians. Twenty technicians actually participated in the study.

In the same way focus group interviews were carried out with a random sample of pharmacists. The names and addresses of 90 pharmacists, 30 from each region were drawn from a database of pharmacists. Eventually eighteen pharmacists participated in the study.

The pharmacists and technicians were invited for the interviews by mail and reminded by phone. The reasons for not participating were: not interested, having to work/time problems, having another appointment and the interview location is too far away.

On average, the group interviews took two hours each and were carried out by two experienced interviewers. The interviews were tape-recorded and written down. Responses to the questions were 
summarised by two researchers, independent from each other. The responses were classified into one of the stages of Rogers' Innovations in Organisations model [1]. After summarising the responses, the summaries were compared and on parts that did not correspond to each other, consensus was reached on the contents of the summary.

\section{Results}

In this section, facilitators and barriers towards implementation of patient education are described as they were reported in the explorative focus group interviews. The data from the focus group interviews were summarised and classified into Rogers' implementation stages. These are redefining/restructuring, clarifying and routinizing.

\subsection{Facilitators}

Table 1 shows the reported, realised patient education activities and preconditions in community pharmacy. These activities and preconditions were classified into the different stages and may be viewed as facilitators for the implementation process.

As can be seen in Table 1, the pharmacy work- process is restructured to facilitate patient education, mainly indicated by realised activities like task division among technicians, attention for special patient groups (like diabetes and hypertension patients) and using a leaflet system to make it easier to give patients extra information. These statements indicate that pharmacies are in the implementation phase.

The pharmacists also reported about the realised patient education at the counter. This was classified into the clarifying stage.

Task division was stated most often in the pharmacists' interviews, whereas attention for special patient groups seems to be important to both pharmacists and technicians. Technicians only stated using a leaflet system. Realised preconditions indicate dealing with restructuring of the pharmacy work-process as well. These include having a patient-friendly pharmacy layout, professionalisation and specialisation with continuing education and motivation of employees, having protocols and the availability of a leaflet system.

In the clarifying stage, only one group of pharmacists mentioned providing patient education. One group of technicians mentioned talking about the way these patient education activities are carried out in staff meetings.

Table 1

Facilitators: realised activities and preconditions for the implementation of patient education ${ }^{\mathrm{a}}$

\begin{tabular}{lll}
\hline Activities and preconditions & $\begin{array}{l}\text { Pharmacists } \\
\text { (three groups) }\end{array}$ & $\begin{array}{l}\text { Technicians } \\
\text { (three groups) }\end{array}$ \\
\hline $\begin{array}{l}\text { Redefining/restructuring stage } \\
\text { Activities }\end{array}$ & $\times \times \times$ & $\times$ \\
$\quad$ Task division & $\times \times$ & $\times \times \times$ \\
$\quad$ Attention for special patient groups & & $\times \times$ \\
Leaflet system is used & $\times$ & $\times$ \\
Preconditions & $\times \times$ & $\times$ \\
$\quad$ Patient-friendly pharmacy lay-out & $\times \times$ & $\times$ \\
Professionalisation of technicians (education) & $\times$ & $\times$ \\
$\quad$ Motivation of technicians & $\times$ & $\times$ \\
Availability of leaflet system & & $\times$ \\
Protocols & & $\times$ \\
Clarifying stage & $\times$ & \\
Pativities & & \\
Preconditions & & $\times$ \\
Patient education is a subject in staff meetings & & $\times$ \\
\hline
\end{tabular}

${ }^{\mathrm{a}}(\times)$ mentioned in one group, $(\times \times)$ mentioned in two groups, $(\times \times \times)$ mentioned in three groups. 


\subsection{Barriers}

The answers to the second question 'What could still be improved with regard to patient education activities?' are shown in Table 2. It is shown that the activities and preconditions are not on a sufficient level for the pharmacists and technicians in the group interviews. They inhibit the improvement of patient education and are therefore seen as barriers for the implementation of patient education.

The replies reveal that pharmacists and technicians have similar ideas regarding the aspects that need improvement and thus serve as barriers. The factors that we classified into the redefining/restructuring stage relate to activities that should be carried out but are not. More specific: technicians' contributions or ideas to improve patient education, technicians' recording (in the computer) of the patient education process with a client and working with a leaflet system. The preconditions that should be improved in this stage include: privacy for the patient, availability of time, availability of patient education protocols, guidance from an external agency (consultant) and a remuneration system that accounts for advice giving.

The main activities to be improved in the clarify- ing stage are providing patient education and repetition, rehearsal of appointments within the pharmacy. The main precondition to be improved in this stage is transfer of knowledge. This means that the information and skills from for instance continuing education courses are used in practice and transferred among technicians but also from pharmacist to technicians. Contributions from technicians, documenting of the patient education process, privacy, protocols, time and giving patient education are particularly important for pharmacists, whereas giving patient education, working with a leaflet system and guidance from an external agency seem to be important for technicians. One of the pharmacists' groups reported they wanted a remuneration system that accounts for advice giving.

\section{Discussion and conclusion}

The facilitators and barriers that emerged from the focus group interviews were classified into two stages in the implementation phase of the diffusion process, the redefining/restructuring stage and the clarifying stage. None of the statements could be classified into the routinizing stage. Thus, it appeared

Table 2

Barriers: activities and preconditions to be improved to facilitate patient education ${ }^{\text {a }}$

\begin{tabular}{llc}
\hline $\begin{array}{l}\text { Activities and preconditions } \\
\text { that need improvement }\end{array}$ & $\begin{array}{l}\text { Pharmacists } \\
\text { (three groups) }\end{array}$ & $\begin{array}{l}\text { Technicians } \\
\text { (three groups) }\end{array}$ \\
\hline $\begin{array}{l}\text { Redefining/restructuring stage } \\
\text { Activities }\end{array}$ & $\times \times \times$ & $\times \times$ \\
$\quad$ Contributions/initiatives from technicians & & $\times \times$ \\
Documenting of the patient education process & & \\
$\quad$ Working with a leaflet system & $\times \times$ & $\times$ \\
Preconditions & $\times \times$ & $\times$ \\
Privacy & $\times \times$ & $\times$ \\
Time to provide patient education & $\times$ & $\times$ \\
Protocols & $\times$ & $\times$ \\
Guidance (from external agency) & & $\times$ \\
Clarifying stage & & $\times$ \\
Activities & $\times \times \times$ & $\times$ \\
Patient education/advice giving & $\times$ & \\
Pepetition of appointments & $\times$ & \\
Knowledge transfer & $\times$ & $\times$ \\
\hline
\end{tabular}

${ }^{\mathrm{a}}(X)$ mentioned in one group, $(X \times)$ mentioned in two groups, $(X \times \times)$ mentioned in three groups. 
that the implementation phase, putting patient education activities into use in daily practice, had not finished.

The reported facilitators in the redefining/restructuring stage were, like the barriers, related to the organisation of patient education activities. The facilitators in the clarifying stage relate to practising of and talking about patient education.

Barriers in the redefining/restructuring stage were reported in the literature as well as in our focus group interviews. In addition to the barriers in the literature (lack of time and privacy) we mainly found barriers related to the organisation of patient education.

In the clarifying stage, pharmacists and technicians were still struggling with practising the learned knowledge and skills, indicated by the reported barriers 'not enough guidance' and 'not enough knowledge transfer'.

The factors that were found as facilitators and barriers seem to be quite alike, but different factors serve either as a barrier or as a facilitator depending on the stage a pharmacy is in. The individual pharmacies do not adopt and implement an innovation at the same time [1]. Therefore, a pharmacy in the routinizing stage might experience a certain factor in the redefining/restructuring stage as facilitating, as realised. On the other hand, a pharmacy that is still in the restructuring stage might experience it as a barrier, it has to be improved.

Some factors, for instance a leaflet system, are reported in more technicians' groups. Other factors, for example task division, initiatives from technicians and protocols, are stated in more pharmacists' groups. This finding shows that pharmacists and technicians have different viewpoints, which is why both groups should be involved in initiatives concerning the improvement of patient education and the evaluation of such projects.

Our conclusion at this point is that patient education is not implemented by Dutch community pharmacies, but efforts are undertaken to proceed and to make patient education an integrated element of the daily activities. The pharmacists and technicians in our group interviews belonged obviously to different adopter categories, because some factors were both stated as 'realised' and 'to be improved'.

It is very likely and most of the times noted that studies were done with pharmacies that belonged to the 'innovators' or 'early adopters', that is about $16 \%$ of the population who are most likely to accept change [1]. Although we tried to obtain a random sample of pharmacists and technicians, due to the high non-response rate we might have obtained a rather selective, motivated sample. However, short interviews with the non-responders showed that they were also involved in patient education activities. We prefer to apply the conclusions of the current explorative study to the innovators and early adopters only. Another remark should be made with regard to the responsiveness of the interviewees. The responses only relate to aspects the interviewed pharmacists and technicians experience as important and that they are aware of. As such, these statements are more or less dependent on the level their pharmacies have reached and on the ability of the respondents to reflect on their work and on their pharmacy.

On the basis of this explorative study, an effect study was designed with a controlled experimental design, with pre- and post-intervention measurement in both the experimental and the control group. The study focuses on the restructuring and clarifying stages, improving the organisation, the work process around patient education activities. The data from this explorative study were used to define the goals and the knowledge and skills needed to achieve these goals. Evaluations are carried out with pharmacists and with technicians.

\section{Acknowledgements}

We acknowledge J.C.H. Bakx, Netherlands Institute for Health Promotion and Disease Prevention (NIGZ), Woerden, The Netherlands and A. Bakker, Department of Pharmacoepidemiology and Pharmacotherapy, Utrecht Institute for Pharmaceutical Sciences (UIPS), The Netherlands.

\section{References}

[1] Rogers EM. Diffusion of innovations, 4th ed, New York: The Free Press, 1995.

[2] Cancrinus-Matthijsse A. Tussen hulpverlening en onder- 
nemerschap. Beroepsuitoefening en takopvattingen van openbare apothekers in een aantal West-Europese landen (Between assistance and entrepreneurschip. Professional practice and task conceptions of community pharmacists in a number of Western European countries. Dissertation, Groningen University, Groningen, 1995.

[3] Van Mil J, De Jong-van den Berg LTW, Tromp TFJ. Pharmaceutical care in the community pharmacy in the Netherlands. Pharm Weekbl (Special Issue) 1998;132:36-9.

[4] Van der Heide H, Tinke JL. Facts and figures 1998: cost development of pharmaceutical aid, s-Gravenhage: Foundation for Pharmaceutical Statistics, 1998.

[5] Blom L. Developing patient education in community pharmacy. Dissertation, University of Utrecht, Utrecht, 1996.

[6] Hepler CD, Strand LM. Opportunities and responsibilities in pharmaceutical care. Am J Hosp Pharm 1990;47:533-43.

[7] Hepler CD. Issues in implementing pharmaceutical care. Am J Hosp Pharm 1993;50:1635-41.

[8] Van de Vaart F. Quality of pharmaceutical care. Pharm Weekbl (Special Issue) 1998;132:31-2.

[9] Moss F. Legislation on medication in the Netherlands. Pharm Weekbl (Special Issue) 1998;132:11-3.

[10] Meade V. OBRA '90: how has pharmacy reacted? Am Pharm 1995;NS35:12-6.

[11] Giuffrida A, Torgersen T. Should we pay the patient? Review of financial incentives to enhance patient compliance. Br Med J 1997;315:703-7.

[12] Herborg H. The pharmaceutical care concept: personal views with a focus on development projects in Denmark. Int Pharm J 1995;9:148-9.

[13] Farris K, Schopflocher DP. Between intention and behavior: an application of community pharmacists' assessment of pharmaceutical care. Soc Sci Med 1999;49:55-66.
[14] Meade V. New services emerge in chain pharmacy. Am Pharm 1993;NS33:23-7.

[15] Blom A, Kam AL, Bakker A, Claesson C. Patient counselling in community pharmacy. A comparative study between Swedish and Dutch pharmacists. J Soc Admin Pharm 1993; 10:53-62.

[16] Tully MP, Hassell K, Noyce PR. Advice giving in community pharmacies in the UK. J Health Serv Res Policy 1997;2:38-50.

[17] Schusler-van Hees M, Essink RTGM, de Smet PAGM. Pharmaceutical patient care in the Netherlands. Pharm Weekbl (Special Issue) 1998;132:33-5.

[18] Burggraaf W. De openbare apotheek als expertisecentrum; Onderzoek naar de mogelijkheden tot optimalisering van de openbare apotheek (Community pharmacy as expertise-centre; research for possibilities to optimise community pharmacy), Breukelen: Management Research Centre Nijenrode, 1995.

[19] Keene JM, Cervetto S. Health promotion in community pharmacy: a qualitative study. Health Educ J 1995;54:28593.

[20] Moore S, Cairns C, Harding G, Craft M. Health promotion in the high street: a study of community pharmacy. Health Educ J 1995;54:272-84.

[21] Perri M, Kotzan J, Pritchard L, Ozburn W, Francisco G. OBRA 90: the impact on pharmacists and patients. Am Pharm 1995;NS35:24-8.

[22] Raisch D. Barriers to providing cognitive services. Am Pharm 1993;NS33:54-8.

[23] Clarke A. Focus group interviews in health-care research. Prof Nurse 1999;14:395-7.

[24] Coreil J. Group interview methods in community health research. Med Anthropol 1995;16:193-210. 